\title{
THE SMALLEST SIZES OF DIFFUSE INTERSTELLAR CLOUDS
}

\author{
John M. Dickey, J. Crovisier and I. Kazes \\ University of Minnesota, Obs. Paris
}

\section{ABSTRACT}

By observing the difference in optical depths between absorption spectra toward the two components of double sources we have measured the variations in opacity over lengths of less than 0.1 up to $10 \mathrm{pc}$ inside diffuse interstellar clouds. Significant variations are detected on scales larger than about 0.2 pc, but not less. This may represent the minimum size for diffuse cloud structure. By comparing the variations of Gaussian fitted line parameters we find that variations in the internal velocity field of diffuse clouds explain the data rather better than tiny independent "cloudlets."

\section{INTRODUCTION}

Interstellar diffuse clouds are difficult to map. In $21 \mathrm{~cm}$ emission it is possible to trace the broad outlines of clouds and cloud complexes (e.g., Verschuur 1974); and with large telescopes it is possible to measure the fluctuations of emission on smaller scales (10' to $1^{\circ}$, e.g., Jahoda et al. 1984), but these fluctuations cannot be interpreted directly as cloud structure because of spatial confusion, self-absorption, and line of sight blending of various regions overlapping in velocity. The ambiguity of higher resolution interferometer emission studies is even greater (e.g., Crovisier and Dickey 1983). Absorption studies at $21 \mathrm{~cm}$ have the drawback that they can only be done in the directions of strong background continuum sources, which are sparse, but they have the great advantage that only the cool gas appears in the spectrum. Since linewidths are narrow for the cool gas there is less velocity blending, and kinematic distance estimates are more accurate than for emission features. Warm gas, which may be associated with the cool cloud (Liszt 1983) does not appear in absorption.

There are two ways to use $21 \mathrm{~cm}$ absorption to study the smaller scale structure of diffuse clouds and cloud complexes. The first is to map the absorption across the face of an extended continuum source. This was first done toward Cas A, Cyg A, and Vir A (Greisen 1973a,b), and it has been improved by many other groups (e.g., Schwarz et a1. 1980, Bregman et a1. 1983, Lockhart and Goss 1978, Landeker et al. 1981, Liszt et al. 1982). In many cases small scale structure is found, but interpretation is difficult because the noise level in absorption usually varies drastically from point to point, and even with the highest resolution interferometers there are seldom more than five to ten independent pixels in either dimension across the source. Gradients in optical depth have been unambiguously identified, and in some cases higher order fluctuations are apparent, but a full statistical characterization of the small scale structure, e.g., the cloud size spectrum or the turbulence spectrum, is not tractable from these isolated regions. The other strategy is to use double sources for the background continuum; if the two components are unresolved such a double gives simply two spectra separated by angle $\theta$. Surveys of absorption toward many such sources are possible with modest telescope and reduction time; the results provide a measure of the average change of optical depth over small angles in diffuse $H$ I clouds. 
A preliminary survey was performed with the NRAO Green Bank interferometer (Dickey 1979); in the present work we have extended this using the WSRT to higher sensitivities and more sources at lower galactic latitudes. Many cases of significant optical depth changes over angles of about $1^{\prime}$ or less have been detected, these correspond to appreciable variations in the column density of cold gas over distances of 0.1 to $10 \mathrm{pc}$. Several clouds are sampled at points separated by less than this distance, but none of them show significant optical depth variations. As discussed below, this is strong evidence that the smallest structures ("cloudlets") in diffuse clouds are at least this large.

\section{OBSERVATIONS}

For background sources for this experiment we have selected extragalactic doubles whose continuum structure was well known, which were at low galactic latitude, with component separations of about $2^{\prime}$ to about $30^{\prime \prime}$. At low latitudes we can use a rotation curve to get kinematic distance estimates for the various spectral features ("clouds") detected. These distances are particularly important for this experiment because they tell us the linear separation between the two lines of sight toward the two components as measured in the absorbing interstellar cloud. If we call the kinematic distance $D$, then this separation is $\mathrm{D} \theta$, where $\theta$ is the angular separation of the two components. For our sample, $D \Theta$ varies from a bit less than $0.1 \mathrm{pc}$ to about $10 \mathrm{pc}$, with most values between 0.2 and $3 \mathrm{pc}$.

The observations were taken at the WSRT in 1981. The velocity channel separation was $1.25 \mathrm{~km} \mathrm{~s}^{-1}$, which we Hanning smoothed to $2.5 \mathrm{~km} \mathrm{~s}^{-1}$ to minimize overlap between adjacent channels. About three hours of integration were spent on each source, the shortest baselines were rejected to eliminate $21 \mathrm{~cm}$ emission which could pollute the absorption spectra. For each velocity channel showing absorption toward both components we computed the difference between the two, and construct a difference spectrum $\Delta t(v)$ for each background source. In this experiment every source shows significant differences in one or more absorption lines. We have also fitted Gaussians to the absorption features toward each component. Data and results in more detail are given by Crovisier et al. (1984).

\section{DISCUSSION}

The structure of an interstellar diffuse cloud may be as complex as a Rorshach figure, and studying maps, no matter how detalled, may be of little value beyond the psychological. The important astrophysical questions relate to the range of sizes of clouds present, the ordered motions and turbulence, and the density, temperature, ionization, and thermodynamic balance. Our results can be used to derive statistics for the small scale structure of clouds in two different ways. These two approaches differ in their assumptions about the way the observed absorption spectra sample the clouds. The first assumes each velocity channel (after Hanning smoothing and resampling) to be a different statistical sample corresponding to a different location inside the cloud. Comparing the corresponding channels of the two spectra gives a sample of the variation of the medium over the distance $D \theta$. This approach gives the distribution shown in the figure, which plots $D \theta$, the linear separation of the two points, vs. the magnitude of the difference in optical depths at the two points. There is a dramatic change in the distribution of the points on the figure between $D \Theta$ less than about $0.2 \mathrm{pc}$ and $\mathrm{D} \Theta$ 
greater than $0.2 \mathrm{pc}$. This change is pronounced even if one deletes several of the highest points, or if one deletes certain background source directions. The implication of the figure is that there is very little variation in optical depth over distances $D \Theta$ of less than about $0.2 \mathrm{pc}$, but that for larger distances the rms change in optical depth quickly increases. We may interpret this as evidence that structures with sizes greater than $0.2 \mathrm{pc}$ are common in interstellar diffuse clouds, but that smaller "cloudlets" are rare.

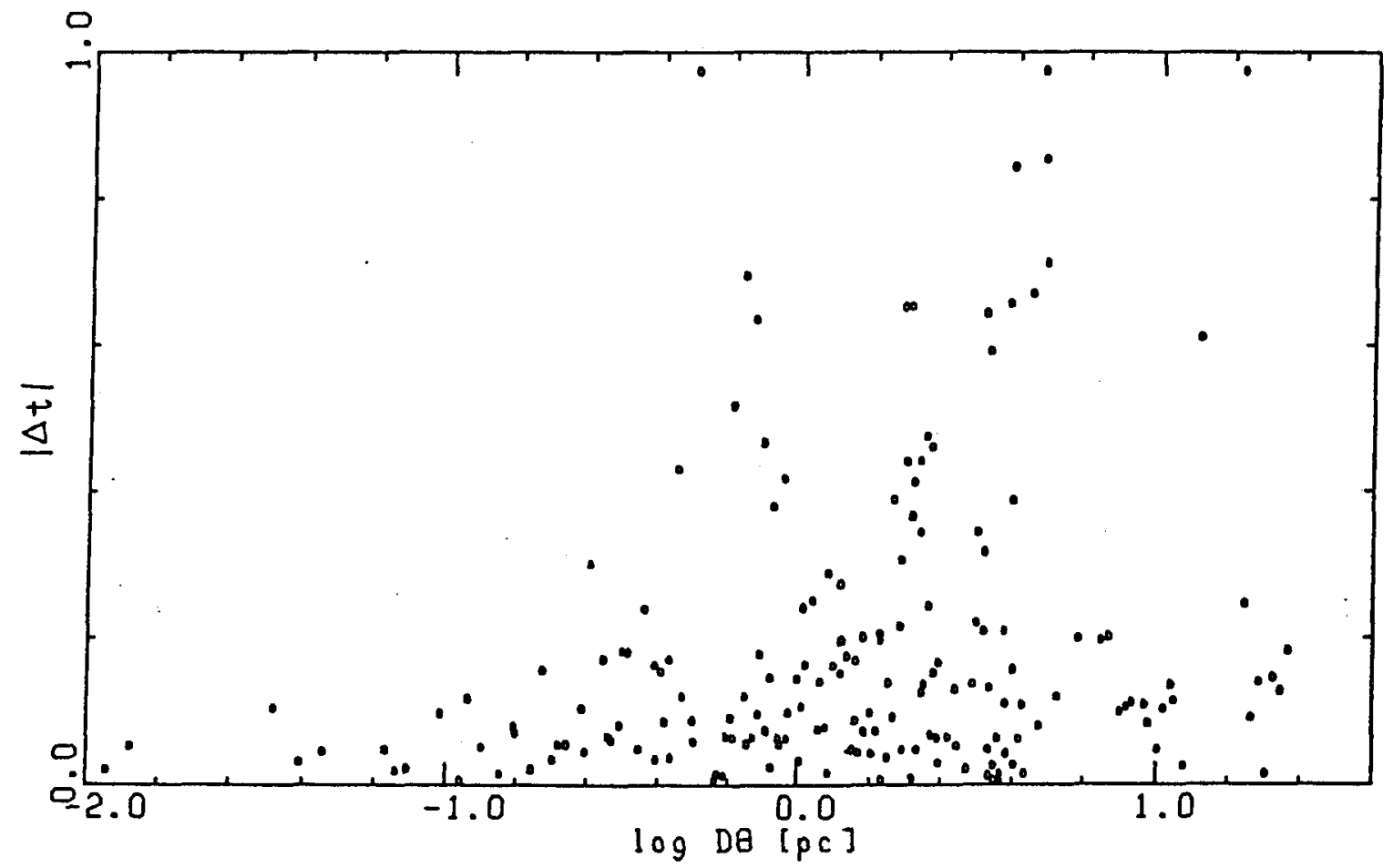

Fig. 1. The distribution of variations in absorption (absolute scale) vs. the linear separation of the two samples $(D \Theta)$. Each point represents one velocity channel for which two optical depths were detected. The abcissa is the difference in relative absorption.

The figure is somewhat deceiving because the points are not necessarily independent. This is not simply because of the velocity resolution, whose effects have been minimized by the Hanning smoothing and resampling, but because of the velocity correlation inherent in the interstellar clouds. It is not correct to assume that each velocity channel is a separate measurement of the fluctuation of optical depth, since the lines are generally Gaussians with widths of several channels. An alternative approach is to look at the variation in the Gaussian fit parameters (1ine depth, center velocity, and velocity width) between the two lines of sight. Each difference gives a measure of the variation of that quantity over the distance $D \Theta$. We can then study separately the increase in variation of line depth with $D \Theta$, line center velocity with $D \Theta$, etc. The increase in variation with $D \Theta$ is still apparent, but the sudden jump at $0.2 \mathrm{pc}$ is no longer so abrupt. In general the increase of variation with $\mathrm{D} \ominus$ is more pronounced for the line center and line width than for the line depth, at least for $D \Theta$ less than about $1 \mathrm{pc}$. This result is highly suggestive that the variations we detect are not due to individual, 
distinct structures with overall sizes of less than one parsec ("cloudlets"), but rather to velocity variations inside larger structures. Whether these variations are due to ordered motion such as rotation, expansion, or collapse (e.g., Goldsmith 1984), or due to random turbulent motion might be tractable If we could measure whether the variation of line center is greater than the variation of line width. This is not yet clear from the data.

If the variations in Gaussian parameters are due to turbulence inside the clouds a parameter of great interest is the turbulence spectrum (Armstrong et al. 1981, Larson 1980, Myers 1983, Dickey 1984). Looking only at the variation in line center velocities between the two lines of sight we find an rms velocity difference of about $1.5 \mathrm{~km} \mathrm{~s}^{-1}$ at $\mathrm{D} \theta$ of 1 to $10 \mathrm{pc}$, vs. $0.5 \mathrm{~km} \mathrm{~s}^{-1}$ at $\mathrm{D} \Theta$ of 0.1 to $1 \mathrm{pc}$. These numbers agree roughly with Myers and Benson's (1984) results for turbulent velocities on the same scales inside molecular clouds, although their measurement used line width observations rather than direct differential absorption observations such as these.

On the other hand if the optical depth variations observed are due to tiny independent cloudlets it is hard to understand why the velocity centers and widths vary more strongly on small scales than the line peaks. It is also difficult to understand how such small clouds could be thermodynamically stable in the interstellar environment. As Cowie and McKee (1977) show, a cloud embedded in the hot phase must be at least a few parsecs in radius to resist evaporation. Smaller clouds might be stable if they were embedded in a classical "inter-cloud medium" of warm H I (Field et al.1969), but the passage of the shock from a young supernova remnant would soon destroy them in a McKeeOstriker (1977) interstellar medium. One possibility is that they are debris from recent cloud collisions (Cowie 1980), and therefore have relatively short ages. But altogether the data at this point seem to suggest a velocity field origin for the small scale structure rather than a cloudlet explanation.

\section{REFERENCES}

Armstrong, J.W., Condes, J.M., and Rickett, B.J., 1981, Nature 291, 561 .

Bregman, J.D., Troland, T.H., Forster, J.R., Schwarz, U.J., Goss, W.M., and Heiles, C., 1983, Astron. Astrophys. 118, 157.

Cowie, L.L. and McKee, C.F., 1977, Ap.J. 211, 135.

Cowie, L.L., 1980, Ap.J. 236, 868.

Crovisier, J. and Dickey, J.M., 1983, Astron. Astrophys. 122, 282.

Crovisier, J., Dickey, J.M. and Kazès, I., 1984, Astron. Astrophys., in preparation.

Dickey, J.M., 1979, Astrophys. J. 233, 558 .

Dickey, J.M., 1984, in IAU Symposium No. 106, The Milky Way, ed. H. van Woerden (Dordrecht: Reidel).

Field, G.B., Goldsmith, D.W., and Habing, H.J., 1969, Ap.J. Lett. 155, L149. Goldsmith, P.F., 1984, Icarus, in press.

Greisen, E.W., 1973, Astrophys. J. 184, 363 and 379.

Jahoda, K., Dickey, J.M., Lockman, F.J., and McCammon, D., 1984, Astrophys. J. submitted.

Landecker, T.L., Roger, R.S., and Higgs, L.A., Astron. Astrophys. Supp1. 39, 133 (1980).

Larson, R.B., 1981, M.N.R.A.S. 194, 809.

Liszt, H.S., Dickey, J.M., and Greisen, E.W., 1982, Ap.J. 261, 102. 
Liszt, H.S., 1983, Ap.J. 275, 163.

Lockhart, I.A. and Goss, W.M., 1978, Astron. Astrophys. 67, 355.

McKee, C.F. and Ostriker, J.P., 1977, Astrophys. J. 218, 148.

Myers, P.C., 1983, Astrophys. J. 270, 105.

Schwarz, U.J., Arnal, E.M., and Goss, W.M., 1980, M.N.R.A.S. 192, 678.

Verschuur, G.L., 1974, Astrophys. J. Supp1. 27, 65.

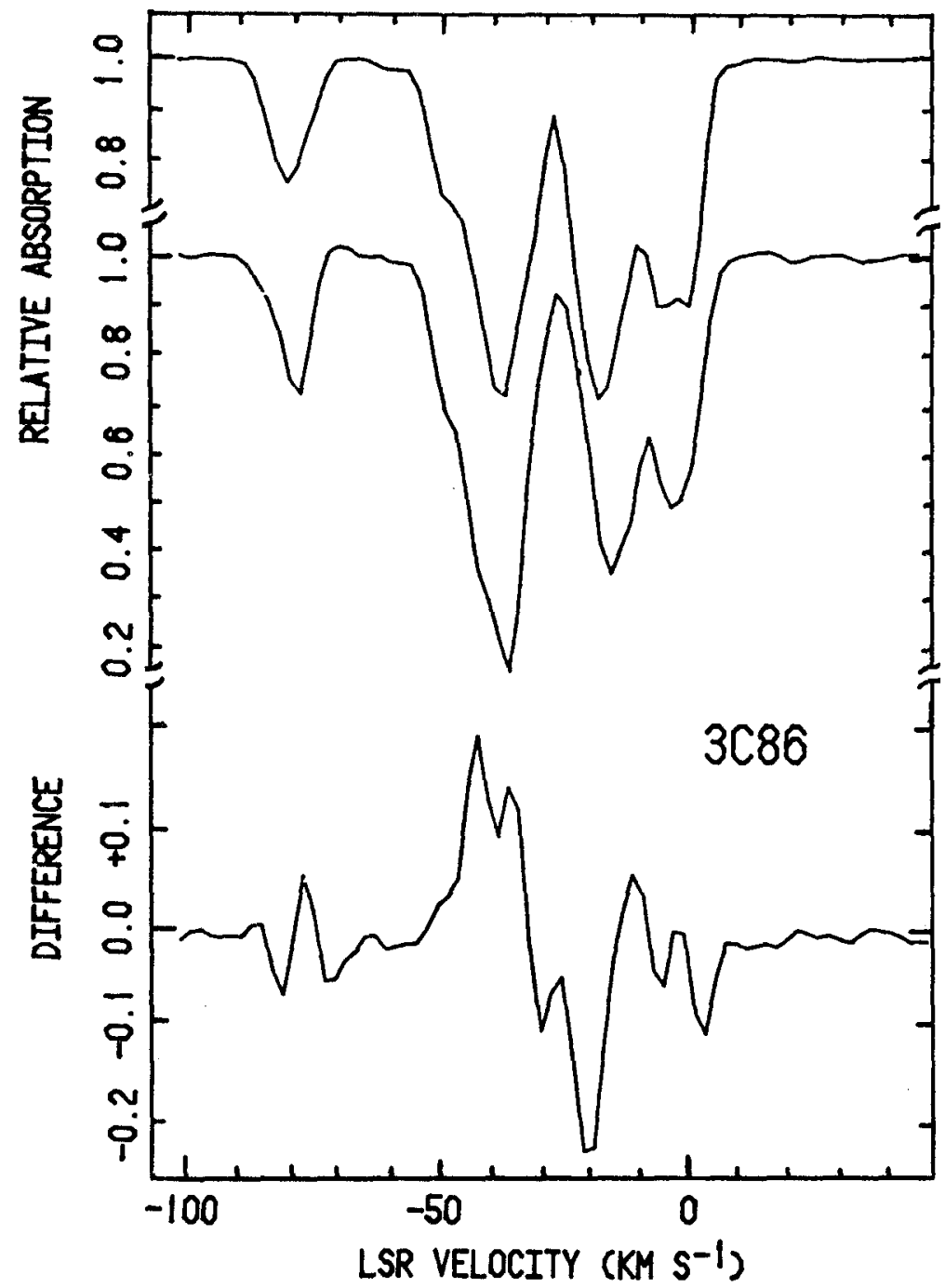

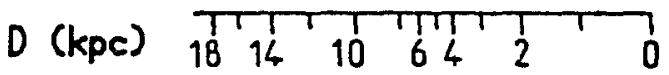

$D \theta$ (pc) 20,1064210

Figure 2. Typical absorption spectra toward 3c86 $(\ell=143.9, b=-1.0)$. The difference spectrum, $\Delta t(v)$ is below. 